\title{
LIPSCHITZIAN MAPPINGS AND TOTAL MEAN CURVATURE OF POLYHEDRAL SURFACES. I
}

\author{
BY
}

\author{
RALPH ALEXANDER
}

\begin{abstract}
For a smooth closed surface $C$ in $E^{3}$ the classical total mean curvature is defined by $M(C)=\frac{1}{2} \int\left(\kappa_{1}+\kappa_{2}\right) d \sigma(p)$, where $\kappa_{1}, \kappa_{2}$ are the principal curvatures at $p$ on $C$. If $C$ is a polyhedral surface, there is a well known discrete version given by $M(C)=\frac{1}{2} \sum l_{i}\left(\pi-\alpha_{i}\right)$, where $l_{i}$ represents edge length and $\alpha_{i}$ the corresponding dihedral angle along the edge. In this article formulas involving differentials of total mean curvature (closely related to the differential formula of L. Schläfli) are applied to several questions concerning Lipschitizian mappings of polyhedral surfaces.

For example, the simplest formula $\sum l_{i} d \alpha_{i}=0$ may be used to show that the remarkable flexible polyhedral spheres of $\mathrm{R}$. Connelly must flex with constant total mean curvature. Related differential formulas are instrumental in showing that if $f$ : $E^{2} \rightarrow E^{2}$ is a distance-increasing function and $K \subset E^{2}$, then $\operatorname{Per}(\operatorname{conv} K) \leqslant$ $\operatorname{Per}(\operatorname{conv} f[K])$.

This article (part I) is mainly concerned with problems in $E^{n}$. In the sequel (part II) related questions in $S^{n}$ and $H^{n}$, as well as $E^{n}$, will be considered.
\end{abstract}

1. Introduction. Suppose there are two pointsets $\left\{p_{0}, p_{1}, \ldots, p_{n}\right\}$ and $\left\{q_{0}, q_{1}, \ldots, q_{n}\right\}$ in a Euclidean space $E^{m}$ which are subject to the Lipschitz condition

$$
\left|q_{i}-q_{j}\right| \leqslant c\left|p_{i}-p_{j}\right| \text { for each pair } i, j \text {. }
$$

There are natural questions of how various geometric functions of the two pointsets are related, if at all. For example, if $m>1$, the condition (1) will imply no general relation between the $m$-volumes of $\operatorname{conv}\left\{q_{1}\right\}$ and $\operatorname{conv}\left\{p_{i}\right\}$, the respective convex covers in $E^{m}$. The set diameter, $\operatorname{Dia}\left\{p_{i}\right\}=\max _{i, j}\left|p_{i}-p_{j}\right|$, provides a simple example of a positively related function in that $\operatorname{Dia}\left\{q_{i}\right\} \leqslant c \operatorname{Dia}\left\{p_{i}\right\}$.

However, there are positive results which are less obvious. If the circumradius of a pointset is defined to be the radius of the least disk which contains the pointset, a theorem of Kirszbraun [9] implies that the circumradii of the two pointsets satisfy $R_{q} \leqslant c R_{q}$. In fact the author has given the following characterization of the circumradius [1]: Let $R$ be the circumradius of the Euclidean pointset $\left\{p_{0}, p_{1}, \ldots, p_{n}\right\}$. Then $2 R^{2}$ equals the maximum of the quadratic form

$$
\sum_{i j}\left|p_{i}-p_{j}\right|^{2} x_{i} x_{j}
$$

subject to the condition that $\left(x_{0}, x_{1}, \ldots, x_{n}\right)$ is a probability vector, i.e., $x_{i} \geqslant 0$ and $\sum_{i} x_{i}=1$.

Received by the editors August 2, 1983 and, in revised form, May 21, 1984. 1980 Mathematics Subject Classification. Primary 52A22; Secondary 53C65.

(C)1985 American Mathematical Society $0002-9947 / 85 \$ 1.00+\$ .25$ per page 
It might be noted that the set diameter and circumstances belong to that class of nonnegative geometric functions $f$ for which (i) $f(K)=f\left(K^{\prime}\right)$ if $K$ is congruent to $K^{\prime}$, (ii) $f(\alpha K)=|\alpha| f(K)$, and (iii) $f$ is continuous with respect to the Hausdorff metric on compact subsets. Many interesting problems have been raised concerning the relationship of the Lipschitz condition (1) to various $f$. Several questions in addition to those treated in the main body will be mentioned at the end of the article.

An important additional property shared by the set diameter and circumradius is (iv) $f(\operatorname{conv} K)=f(K)$. So unless it is stated otherwise, $K$ will always denote a convex, compact subset of Euclidean space.

The present article treats those integral mean curvature functions $M$, or equivalently Quermassintegrale, which satisfy in particular condition (ii) above. Any integral mean curvature function satisfies (i) and (iii), at least when suitably generalized in the manner of Minkowski (as in formula (4) below).

In $E^{1}, M(K)$ is the length of $K$; in $E^{2}, M(K)$ is $\operatorname{Per}(K)$, the perimeter of $K$. For a smooth body in $E^{3}$ the total mean curvature is given by the classical formula

$$
M(K)=\int_{\partial K} \frac{1}{2}\left(\kappa_{1}+\kappa_{2}\right) d \sigma(p)
$$

where the $\kappa_{i}$ are the principal curvatures at the surface point $p_{i}$.

A formula for the integral mean curvature for a smooth body in $E^{m}$ can be given conveniently in terms of an integral over the spherical image:

$$
M(K)=\int_{S^{m-1}}(m-1)^{-1}\left(R_{1}(u)+\cdots+R_{m-1}(u)\right) d \sigma(u)
$$

where $R_{i}=\kappa_{i}^{-1}$, and $S^{m-1}$ denotes the unit $(m-1)$-sphere. It is clear from (3) that $M$ satisfies condition (ii). (See Firey [7] for a solution of the long-standing problem of characterizing those functions $f: S^{n} \rightarrow E^{1}$ which can be represented as $f(u)=$ $R_{1}(u)+\cdots+R_{n-1}(u)$ for some convex body $K$ in $E^{n}$.)

A major advance in integral geometry was achieved by Minkowski when he obtained the famous formula

$$
M(K)=\int_{S^{m-1}} H(u) d \sigma(u)
$$

where $H(u)=\max \{\langle x, u\rangle: x \in K\}$ is the usual support function for $K$. An important feature of the formula (4) is that it defines the function $M$ for any bounded convex set in $E^{m}$. Also, it is easily seen from (4) that $M$ is continuous with respect to the Blaschke-Hausdorff metric on compact convex subsets of $E^{m}$.

There exists yet another fruitful interpretation of the number $M(K)$. There is a motion-invariant measure on the oriented hyperplanes of $E^{m}$ (a Crofton measure). The space of oriented planes may be coordinatized by the points on the cylinder $S^{m-1} \times \mathbf{R}$. The point $(u, r), r>0$, is associated with the plane of distance $r$ from 0 and positive outer normal $u ;(-u,-r)$ is associated with the plane if the inner normal is positive. The $\eta$-measure of a planeset is the usual cylindrical measure of the coordinate pointset. The following formula can be deduced rather easily from (4):

$$
M(K)=\frac{1}{2} \eta\{h: h \cap K \neq \varnothing\} .
$$


One goal of the present article is to prove the following result.

THEOREM 1. Let the pointsets $\left\{p_{0}, p_{1}, \ldots, p_{n}\right\}$ and $\left\{q_{0}, q_{1}, \ldots, q_{n}\right\}$ in $E^{m}$ satisfy the Lipschitz condition (1). Then

$$
M\left(\operatorname{conv}\left\{q_{i}\right\}\right) \leqslant c M\left(\operatorname{conv}\left\{p_{i}\right\}\right) .
$$

In the sequel (Part II) we shall give an integral geometric proof of Schläfli's differential formula (which is briefly stated in \$9), and give yet another proof of Theorem 1.

Since in $E^{2}, M(K)=\operatorname{Per}(K)$, we wish to state a corollary theorem which is of independent interest. In fact it answers the original question which motivated the present work.

THEOREM 2. Let the planar pointsets $\left\{p_{0}, p_{1}, \ldots, p_{n}\right\}$ and $\left\{q_{0}, q_{1}, \ldots, q_{n}\right\}$ satisfy the Lipschitz condition (1). Then

$$
\operatorname{Per}\left(\operatorname{conv}\left\{q_{i}\right\}\right) \leqslant c \operatorname{Per}\left(\operatorname{conv}\left\{\dot{p_{i}}\right\}\right) .
$$

A basic result on a differential invariant involving the edges and dual-dihedral angles of a deformed simplex (Theorem 4) leads at once to Theorem 1. Before proceeding, we mention at least one unexpected by-product.

Recently, Connelly [6] has made the remarkable discovery of embedded polyhedral 2-spheres in $E^{3}$ which are not rigid. Such surfaces, which are the only known examples of embedded closed polyhedral surfaces that flex, are termed Connelly spheres. The question of whether or not a Connelly sphere must flex with constant volume has been raised (the bellows conjecture). Since the derivative formulas add properly under simplicial decomposition in $E^{3}$, we can at least obtain the following theorem.

THEOREM 3. A Connelly sphere flexes with constant total mean curvature.

In addition to the proof using the principal method of this paper, two other methods of proof for Theorem 3 are given in $\$ 9$. In fact, if the reader wishes a direct and elementary proof of Theorem 3 , this may be obtained by first reading $\S 3$, and then going to $\$ 9$ for a direct proof of equation (11) valid for dimension $n=3$. The application to Connelly spheres is explained in a self-contained manner in $\$ 7$.

In this paper the invariant measure $\eta$ on the hyperplanes of $E^{n}$ plays an important role, and the arguments involving $\eta$ are essentially complete and self-contained. However, much of what is done can be generalized to $\eta_{r}$, the invariant measure on the $r$-flats of $E^{n}$. Even though a thorough discussion of $\eta_{r}$ is not included (complete information may be found in Santaló's book [11]), an extended version of the basic differential invariant is outlined in $§ 8$.

The principal method of the paper exploits the fact that the first derivative of the angular measure of a varying angle may be recovered from orthogonal projections (Theorem 5). Apologies are offered in advance for the somewhat involved proof; we were not able to find results of this type in the literature. It is hoped that our general approach in this article will give still more information on questions of rigidity and how this concept is related to mean curvature and perhaps to higher order curvatures as well. 
2. Moving points in Euclidean space. Let $\left\{p_{0}, p_{1}, \ldots, p_{n}\right\}$ and $\left\{q_{0}, q_{1}, \ldots, q_{n}\right\}$ be pointsets in $E^{m}$. For $0 \leqslant t \leqslant 1$ we define $p_{i}(t)$ in $E^{2 m}$ by

$$
p_{i}(t)=\left(\sqrt{1-t} p_{i}, \sqrt{t} q_{i}\right) \text {. }
$$

It is clear that $p_{i}(t)$ is a smooth $\left(C^{\infty}\right)$ path in $E^{2 m}$ for $0<t<1$, and that for each pair $i, j,\left|p_{i}(t)-p_{j}(t)\right|$ is a monotone function in $t$.

Now suppose that $m=n$, and that the pointsets $\left\{p_{i}\right\}$ and $\left\{q_{i}\right\}$ are the respective vertex sets of a pair of nondegenerate simplices; in addition assume that $p_{0}=q_{0}=0$. Let $\left[\left\langle p_{i}(t), p_{j}(t)\right\rangle\right], 1 \leqslant i, j \leqslant n$, denote the $n \times n$ Gramian matrix of inner products. Note that

$$
\left[\left\langle p_{i}(t), p_{j}(t)\right\rangle\right]=(1-t)\left[\left\langle p_{i}, p_{j}\right\rangle\right]+t\left[\left\langle q_{i}, q_{j}\right\rangle\right] .
$$

The equation (9) shows that for $0 \leqslant t \leqslant 1$, the matrix $\left[\left\langle p_{i}(t), p_{j}(t)\right\rangle\right]$ is positive definite since each of the two matrices on the right side of (9) is positive definite. Hence the points $\left\{p_{i}(t)\right\}$ are the vertices of a nondegenerate $n$-simplex in $E^{2 n}$. However, by choosing a smoothly changing reference frame, we may assume the homotopy $\left\{p_{i}(t)\right\}$ occurs in a fixed copy of $E^{n}$. We summarize these observations in the following lemma.

Lemma 1. Let $\left\{p_{0}, p_{1}, \ldots, p_{n}\right\}$ and $\left\{q_{0}, q_{1}, \ldots, q_{n}\right\}$ be the respective vertex sets of two nondegenerate simplices in $E^{n}$. Then there is a piecewise smooth homotopy $\left\{p_{0}(t), p_{1}(t), \ldots, p_{n}(t)\right\}$ in $E^{n}$ such that: (i) $\left|p_{i}(0)-p_{j}(0)\right|=\left|p_{i}-p_{j}\right|$ and $\left|p_{i}(1)-p_{j}(1)\right|=\left|q_{i}-q_{j}\right|$ for each pair $i, j$; (ii) for each $t,\left\{p_{i}(t)\right\}$ is the vertex set of a nondegenerate simplex; (iii) for each pair $i, j,\left|p_{i}(t)-p_{j}(t)\right|$ is monotone in $t$.

The homotopy (8) seems to be a very effective choice for the type of problem considered in this article. For example, if the pointsets $\left\{p_{i}\right\}$ and $\left\{q_{i}\right\}$ both lie on a sphere of radius $R$, so does the pointset $\left\{p_{i}(t)\right\}$; and hence the homotopy may be applied to metric inequalities on the sphere. A suitable modification also applies to hyperbolic space. A strengthened version of the lemma, where for any $t, p_{i}(t)$ is moving for at most one value of $i$, can be proved via a compactness argument. This refinement is useful for certain problems, but is unnecessary for the present work.

3. The total mean curvature of a simplex. Before proceeding, it is necessary to introduce notation. If $\left\{p_{0}, p_{1}, \ldots, p_{n}\right\}$ is the vertex set of a nondegenerate simplex $K$ in $E^{n}$, let $e_{i j}$ denote the segment joining $p_{i}$ and $p_{j}$, and let $l_{i j}$ denote the length of $e_{i j}$.

Suppose that $h_{0}$ is a hyperplane, orthogonal to $e_{i j}$, which cuts the interior of $e_{i j}$ at a point $q$. Now $h_{0} \cap K$ is a convex $(n-1)$-polytope which possesses a certain discrete Gaussian curvature $\beta_{i j}$ in $h_{0}$ at the vertex $q$. The number $\beta_{i j}$, which does not depend on the choice of $h_{0}$, will be called the curvature of the edge $e_{i j}$.

The following formula for $M(K)$ is known [11, Chapter 13]:

$$
M(K)=c_{n} \sum_{i j} \beta_{i j} l_{i j},
$$

where $c_{n}$ is a dimensional constant. There are various ways to prove (10), and at least one method will appear later in this article. 
Next, suppose that $K(t), a<t<b$, is a collection of nondegenerate simplices in $E^{n}$ determined by a smoothly varying vertex set $\left\{p_{0}(t), p_{1}(t), \ldots, p_{n}(t)\right\}$. Such a collection will be called smooth. The homotopy discussed in $\$ 2$ provides a useful example of a smooth collection of simplices.

It is now possible to state a theorem on the derivative of total mean curvature. A major portion of this article is devoted to its proof. (See $\S 8$ for an extended version.)

THEOREM 4. Let $l_{i j}(t)$ and $\beta_{i j}(t)$ be the associated edge length and edge curvature function for a smooth collection $K(t)$ of simplices in $E^{n}$. Then

$$
\sum_{i j} l_{i j}(t) d / d t \beta_{i j}(t)=0,
$$

and hence

$$
d / d t M(K(t))=c_{n} \sum_{i j} \beta_{i j}(t) d / d t l_{i j}(t)
$$

Throughout the article, the smoothness of $\beta_{i j}(t)$ and certain related angle measures is assumed without proof. For general information on the structure of convex polyhedra, see the book [8] of Grünbaum.

4. A dynamic formula in integral geometry. The possibility of using integral geometry to study derivatives (or differentials) of geometric functions such as $M$ appears to have received little attention. Therefore it seems necessary to develop from first principles several ideas to be used later.

In the classical development of integral geometry, fundamental notions such as Quermassintegrale, kinematic formula, etc., involve integrals of bounded functions; and hence the standard theorems of integration may be directly applied. This luxury does not exist in the type of integral formula discussed in this section. There seem to be many interesting problems in the general area, but we focus our attention and ignore many obvious questions of generalization.

An angle $A$ is defined to be a closed, convex, polyhedral cone in $E^{n}$. If $A$ is contained in a $k$-plane, $A$ is called a $k$-angle. The $k$-measure $\alpha A$ of a $k$-angle is defined to be $\sigma^{(k-1)} A^{\prime}$, where $A^{\prime}=A \cap S^{n-1}$, and $\sigma^{(k-1)}$ is $(k-1)$-measure on the unit sphere $S^{n-1}$, which is understood to be centered at the vertex of $A$. The facets of an angle will be termed the sides of the angle.

The angle $A$ is determined by a vertex $v$ and unit vectors $u_{1}, u_{2}, \ldots, u_{r}$ which direct the extreme rays of $A$. We wish to define a smooth family of angles $A(t), a<t<b$, as the family given by the smooth vector functions $v(t), u_{1}(t), \ldots, u_{r}(t)$. It will be understood that in the interval $a<t<b$, the unit vector functions $\left\{u_{i}(t)\right\}$ direct the exact set of extreme rays.

For the purposes of this paper it may be assumed that $k=n-1$ and that $r=n-1$, the least number for which $\alpha A$ could be positive.

Let $A(t)$ be a smooth collection of $(n-1)$-angles; and for the arbitrary point $q$ in $E^{n}$, let $H(q)$ denote the set of oriented planes which pass through $q$. There is an obvious invariant measure $\mu$ on $H(q)$, obtained by identifying each oriented plane $h$ via its positive unit normal $n(h)$ at $q$ with a point on $S^{n-1}$. Put $\mu(H(q))=\sigma_{n-1}$, the 
usual measure of $S^{n-1}$, for normalization. Let $A(h, t)$ be the $(n-1)$-angle obtained by projecting $A(t)$ orthogonally into $h$.

The remainder of the section is devoted to the proof of the following theorem. Throughout the paper $c_{n}, d_{n}$, etc., will denote general dimensional constants depending only on $n$. The same symbols might denote other such constants later.

THEOREM 5. If $A(t), a<t<b$, is a smooth collection of $(n-1)$-angles in $E^{n}$, then

$$
d / d t \alpha A(t)=c_{n} \int_{H(q)} d / d t \alpha A(h, t) d \mu(h) .
$$

Before beginning the proof, some remarks are in order. The difficulty revolves about the fact that $d / d t \alpha A(h, t)$ generally possesses unbounded singularities where $n(h)$ is parallel to an extreme ray of $A(t)$. Consideration of the simplest situation of a smooth collection of 2-angles of $E^{3}$ makes this clear. While formula (14) seems natural, there seems to be no simple justification. In fact it will be shown in $\$ 10$ that no corresponding formula exists for $d^{2} / d t^{2} \alpha A(t)$.

Standard ideas of invariant measures in integral geometry show that for each $t$,

$$
\alpha A(t)=c_{n} \int_{H(q)} \alpha A(h, t) d \mu(h) .
$$

It follows at once from (15) that

$$
d / d t \alpha A(t)=\operatorname{limit}_{\Delta t \rightarrow 0} c_{n} \int_{H(q)} \Delta t^{-1}[\alpha A(h, t+\Delta t)-\alpha A(h, t)] d \mu(t) .
$$

Let $\left\{\Delta_{i} t\right\}$ be a sequence of positive numbers such that $a<t+\Delta_{i} t<b$ for each $i$, and $\sum_{i} \Delta_{i} t$ converges. If it is verified that (16) implies (14) as $\Delta t$ tends to zero over such a sequence, then the general validity of (14) follows at once. This is because any sequence $\left\{\Delta_{j} t\right\}$ of nonzero numbers tending to zero contains a subsequence $\left\{\Delta_{i} t\right\}$ such that all terms are of the same sign and $\sum_{i} \Delta_{j} t$ converges.

Before proceeding further, the translation invariance in formula (15) allows several simplifying assumptions which cause no loss of generality. First, since the vertex velocity vector $d / d t v(t)$ makes no contribution to the integral in (14), it may be assumed that $v(t)$ is constant. Second, since the location of $q$ does not influence (14), (15), or (16), it may be assumed that $v=q$.

Also, it will be understood that any sphere mentioned in the remainder of this section will be centered at $q$. For convenience, if $B$ is a subset of $E^{n}$, let $B^{\prime}$ denote $B \cap S^{n-1}$.

The Lebesgue dominated convergence theorem will be used to show that the limit may be taken inside the integral in (16), and thereby establish (14). In order to accomplish this it seems necessary to obtain a tractible bound for

$$
\left|\Delta_{i} \alpha A(h, t)\right|=\left|\alpha A\left(h, t+\Delta_{i} t\right)-\alpha A(h, t)\right| .
$$

The following lemma provides a suitable estimate.

Lemma 2. Let $n_{1}, n_{2}, \ldots, n_{s}$ be the normals to the sides of a $k$-angle $A$ in $E^{k}$. Suppose the angle is perturbed. Then

$$
|\Delta \alpha A| \leqslant d_{k} \sum_{j=1}^{s}\left|\Delta n_{j}\right| .
$$


Proof. The lemma is easily seen to be true when $k=2$ (and $s=2$, necessarily). The general inequality (17) will be established via a covering argument. We may assume that the vertex remains fixed.

Let $h_{1}, h_{2}, \ldots, h_{s}$ be the $(k-1)$-planes which determine the sides of $A$, and let $\bar{A}$ be the result of perturbation. Note that for each $j, \bar{h}_{j} \cap S^{k-1}$ is contained in a slab $W_{j}$ of width $2\left|\Delta n_{j}\right|$ centered by $h_{j}$. Thus

$$
\alpha \bar{A} \leqslant \alpha A+\sum_{j=1}^{s} \sigma^{(k-1)} W_{j}^{\prime} ;
$$

and since

$$
\sigma^{(k-1)} W_{j}^{\prime} \leqslant 2 \pi \sigma_{k-2}\left|\Delta_{j} n\right|
$$

it follows that

$$
\alpha \bar{A}-\alpha A \leqslant d_{k} \sum_{j=1}^{s}\left|\Delta_{j} n\right| .
$$

The reverse inequality is obtained by merely reversing the roles of $A$ and $\bar{A}$ while noting that $\left|-\Delta n_{j}\right|=\left|\Delta n_{j}\right|$. This proves the lemma.

It would be improper to employ, as one could easily do, more precise results such as Schläfli's formula (equation (41) in \$9) in the proof of the lemma because this would invalidate the application of Theorem 5 to such formulas.

The lemma allows $|\Delta \alpha A(h, t)|$ to be estimated by means of a careful examination of $\left|\Delta n_{j}(h, t)\right|, j=1,2, \ldots, s$. To this end let $S^{n-1}$ lie in $E^{n}$, and $h$ be an $(n-1)$-plane through $q$. If $p, p \neq \pm n(h)$, lies on $S^{n-1}$, the polar projection mapping $\Theta_{h}$ onto the $(n-2)$-sphere $h^{\prime}$ is defined by letting $\Theta_{h}(p)$ be the unique point on $h^{\prime}$ where the great circle arc from $n(h)$ through $p$ (the meridian through $p$ ) strikes $h^{\prime}$.

If $S^{k}, k \leqslant n-2$, is a great sphere of $S^{n-1}$ not containing $n(h)$, then $\Theta_{h}\left[S^{k}\right]$ is a great $k$-sphere lying in $h^{\prime}$. Balancing this helpful property is the fact that $\Theta_{h}$ is not an isometry. The mapping $\Theta_{h}$ is of central importance in the study of formula (14) because if $A(t)$ is an $(n-1)$-angle, not containing $\pm n(h)$, then

$$
\alpha A(h, t)=\sigma^{(n-2)}\left(\Theta_{h}\left[A^{\prime}(t)\right]\right) .
$$

The pointsets $A^{\prime}(t)$ and $A^{\prime}(h, t)$ are spherical $(n-2)$-polytopes. If $A^{\prime}(t)$ has $s$ sides, then so will $A^{\prime}(h, t)$. The sides of $A^{\prime}(t)$ will be determined by a collection $S_{1}^{n-3}(t), \ldots, S_{s}^{n-3}(t)$ of $(n-3)$-spheres which are mapped by $\Theta_{h}$ onto a collection of $(n-3)$-spheres $S_{1}^{n-3}(h, t), \ldots, S_{s}^{n-3}(h, t)$ lying in the $(n-2)$-sphere $h^{\prime}$. When $n=3$ it is understood that an $(n-3)$-sphere is a pair of antipodal points on $S^{2}$. (For the purposes of this paper it may be assumed that $s=n-1$.)

Next suppose that $S^{n-3}(t), a<t<b$, is a smooth collection of $(n-3)$-spheres on $S^{n-1}$, none of which contain $n(h)$, i.e., a collection determined by a smooth path on the Grassmann manifold of $(n-2)$-planes through $q$. Put $S^{n-3}(h, t)=$ $\Theta_{h}\left[S^{n-3}(t)\right]$.

At each time $t$ the motions of $S^{n-3}(t)$ and $S^{n-3}(h, t)$ determine two vector fields, $V(t)$ and $V(h, t)$, respectively. The velocity at each point gives a vector and, taken together, these vectors define $V(t)$ and $V(h, t)$, which are special cases of Jacobi 
vector fields. It is clear that these fields are tangent to $S^{n-1}$. Also, it is convenient to define the vector field $N(h, t)$ by taking at each point of $S^{n-3}(h, t)$ the component of $V(h, t)$ which is normal to $S^{n-3}(h, t)$.

If $W$ is a vector field, put $|W|=\sup \{|x|: x \in W\}$. Choose $M$ so that $|V(t)| \leqslant M$ for $a \leqslant t \leqslant b$. Compactness shows that such an $M$ exists since $|V(t)|$ is a continuous function of $t$.

If $\rho(h, t)$ is the mimimum distance between $n(h)$ and points of $S^{n-3}(t)$, the following vital inequality holds

$$
|V(h, t)| \leqslant 2 M \rho(h, t)^{-1} .
$$

The inequality (19) may be validated by observing that if $p$ is on $S^{n-3}(t), \Theta_{h}(p)$ may be calculated by first projecting $p$ orthogonally to $p^{*}$ on $h$ and then projecting $p^{*}$ radially to $\Theta_{h}(p)$ on $h^{\prime}$. If $d p / d t$ is the velocity of $p$, it is seen that $(d / d t) \Theta_{h}(p)$ $=\delta^{-1}\left(d p^{*} / d t\right)_{0}$ where $\delta=\left|q-p^{*}\right|$ and $\left(d p^{*} / d t\right)_{0}$ is the component of $d p^{*} / d t$ which is normal to the vector $q-p^{*}$. Since $\left|\left(d p^{*} / d t\right)_{0}\right| \leqslant|d p / d t| \leqslant M$ and $\delta$ $\geqslant \frac{1}{2} \rho(h, t)$, the inequality (19) follows.

Because of the dimensional gap of 2 between $(n-1)$ and $(n-3)$ we have

$$
\int_{H(q)} \rho(h, t)^{-1} d \mu(h)<\infty .
$$

Since $H(q)$ is compact the integrability of $\rho^{-1}$ is equivalent to local integrability. That $\rho^{-1}$ is locally integrable is established in essentially the same manner as showing $|x|^{-1}$ is locally integrable in $E^{2}$.

The vector field $N(h, t)$, introduced above, for the smooth famly $S^{n-3}(h, t)$ in the $(n-2)$-sphere $h^{\prime}$ has an especially pretty structure which is easy to visualize. The field $V(h, t)$ is certainly tangent to $h^{\prime}$, hence the field $N(h, t)$ of components normal to $S^{n-3}(h, t)$ is also. It is seen that there are antipodal points $\pm \bar{p}$ on $S^{n-3}(h, t)$ such that $\left|d \bar{p} / d t_{N}\right|=\left|d(-\bar{p}) / d t_{N}\right|=|N(h, t)|$, while on the equatorial $(n-4)$-sphere for $\pm \bar{p}$ on $S^{n-3}(h, t)$ we have $d p / d t_{N}=0$; here, $x_{N}$ is the $N$-component of $x$.

Next, let $n(h, t)$ be the positive pole to $S^{n-3}(h, t)$ on the $(n-2)$-sphere $h^{\prime}$. Observe that $\pm d / d t n(h, t)= \pm(d \bar{p} / d t)_{N}$ so that

$$
|N(h, t)|=\left|\frac{d}{d t} n(h, t)\right| \leqslant 2 M \rho(h, t)^{-1} .
$$

The inequality (21) uses (19) and the obvious fact that $|N(h, t)| \leqslant|V(h, t)|$. While (21) shows that $|(d / d t) n(h, t)|$ is $\mu$-integrable, still more calculation is necessary.

If $a<t+\Delta t<b$, then $S^{n-3}(t+\Delta t)$ is contained in a tubular neighborhood $\mathscr{J}$ of radius $M|\Delta t|$ about $S^{n-3}(t)$; or equivalently, the Hausdorff distance between these spheres will not exceed $M|\Delta t|$.

Define $F_{i}(h)=2 / \Delta_{i} t$ if $n(h)$ is in the tubular neighborhood $\mathscr{J}_{i}$ of radius $2 M\left|\Delta_{i} t\right|$ about $S^{n-3}(t)$, and if $n(h)$ lies outside $\mathscr{J}_{i}$ define $F_{i}(h)=4 M \rho(h, t)^{-1}$. Here the sequence $\left\{\Delta_{i} t\right\}$ and the function $\rho$ are as defined above.

Now

$$
\left|n\left(h, t+\Delta_{i} t\right)-n(h, t)\right| \leqslant \int_{0}^{\Delta_{i} t}\left|\frac{d}{d t} n(h, t+y)\right| d y \leqslant 2 M \int_{0}^{\Delta_{i} t} \rho(h, t+y)^{-1} d y .
$$


For any $y$ satisfying $0 \leqslant y \leqslant \Delta_{i} t$, each point of $S^{n-3}(h, t+y)$ is at least $M \Delta_{i} t$ distant from the boundary of $\mathscr{J}_{i}$ and, of course, interior to $\mathscr{J}_{i}$. Thus, if $n(h)$ lies outside of $\mathscr{J}_{i}, \rho(h, t+y) \geqslant M \Delta_{i} t$. Also, it is always true that

$$
\left|\left(h, t+y_{1}\right)-\rho\left(h, t+y_{2}\right)\right| \leqslant M\left|y_{1}-y_{2}\right| \leqslant M \Delta_{i} t .
$$

We conclude that if $0 \leqslant y \leqslant \Delta_{i} t$ and $n(h)$ lies outside $\mathscr{J}_{i}$, then

$$
\rho(h, t) \leqslant 2 \rho(h, t+y) \text {. }
$$

Thus in this situation

$$
2 M \int_{0}^{\Delta_{i} t} \rho(h, t+y)^{-1} d y \leqslant 4 M \int_{0}^{\Delta_{i} t} \rho(h, t)^{-1} d y=4 M \Delta_{i} t \rho(h, t)^{-1},
$$

and hence $\Delta_{i} t^{-1}\left|n\left(h, t+\Delta_{i} t\right)-n(h, t)\right| \leqslant 4 M \rho(h, t)^{-1}$.

The previous work shows that for each $i$, the difference quotient $\Delta_{i} t^{-1}\left|\Delta_{i} n(h, t)\right|$ is dominated by $F_{i}(h)$. Now

$$
\int_{H(q)} F_{i}(h) d \mu(h) \leqslant \int_{\mathscr{F}_{i}} \frac{2}{\Delta_{i} t} d \mu(h)+\int_{H(q)} 4 M \rho(h, t)^{-1} d \mu(h) .
$$

The second integral is over those $h$ for which $n(h)$ lies in $\mathscr{J}_{i}{ }^{\prime}$.

The rightmost integral in (22) is finite by (20). The area of $\mathscr{J}_{i}^{\prime}$ is less than $16 M^{2} \sigma_{n-3} \Delta_{i} t^{2}$; so the second integral in (22) is less than $32 M^{2} \sigma_{n-3} \Delta_{i} t$.

Next set $G(h)=\max _{i} F_{i}(h)$. It follows by summing (22) over $i$ that

$$
\int_{H(q)} G(h) d \mu(h) \leqslant 32 M^{2} \sigma_{n-3} \sum_{i} \Delta_{i} t+4 M \int_{H(q)} \rho(h, t)^{-1} d \mu(h) .
$$

Thus $G(h)$ is integrable and dominates the sequence of difference quotients $\left\{\Delta_{i} t^{-1}\left|\Delta_{i} n(h, t)\right|\right\}$.

Finally, we consider the inequalities

$$
\left|\Delta_{i} \alpha A(h, t)\right| \leqslant d_{n-1} \sum_{j=1}^{s}\left|\Delta_{i} n_{j}(h, t)\right| \leqslant d_{n-1} \Delta_{i} t \sum_{j=1}^{s} G_{j}(h) .
$$

The first inequality of (23) is obtained by applying Lemma 2 to $A(h, t)$. The second inequality is obtained by constructing $G_{j}(h)$ to dominate the sequence $\left\{\Delta_{i} t^{-1}\left|\Delta_{i} n_{j}(h, t)\right|\right\}, j=1, \ldots, s$, in the manner described above. The $\mu$-integrable function $d_{n-1} \sum_{j} G_{j}$ allows the dominated convergence theorem to be applied to (16). This completes the proof of Theorem 5 .

5. A proof of Theorem 4. Let $K(t), a<t<b$, be a smooth family of nondegenerate simplices in $E^{n}$. If for a fixed $t$, the hyperplane $h$ cuts the simplex $K(t)$ so that no vertex $p_{i}(t)$ lies on $h$, we say that $h$ properly cuts $K(t)$. Here $h \cap K(t)$ is an $(n-1)$-polytope whose vertices lie in the relative interiors of various edges of $K(t)$. If $q$ is such a vertex lying in the edge $e_{i j}(t)$, we define $\bar{\gamma}_{i j}(h, t)$ to be the discrete Gaussian curvature in $h$ at $q$ for the $(n-1)$-polytope $h \cap K(t)$.

If the plane $h$ does not cut $K(t)$ properly, define $\gamma_{i j}(h, t)=0$ for all $i, j$. Otherwise, define $\gamma_{i j}(h, t)=0$ for all pairs $i, j$ where $h$ does not cut $e_{i j}(t)$, and define $\gamma_{i j}(h, t)=\bar{\gamma}_{i j}(h, t)$ if $h$ cuts $e_{i j}(t)$. 
Since proper cutting is an open condition with respect to $t$, we have the following formulas for a hyperplane $h$ which properly cuts $K(t)$;

$$
\begin{aligned}
& \sum_{i j} \gamma_{i j}(h, t)=\sigma_{n-2}, \\
& \sum_{i j} \frac{d}{d t} \gamma_{i j}(h, t)=0 .
\end{aligned}
$$

We wish to integrate equations (24) and (25) term by term with respect to the Crofton measure $\eta$ on the hyperplanes of $E^{n}$. At present we are unable to perform any meaningful integration of the corresponding equations for second and higher derivatives of $\gamma_{i j}(h, t)$.

Suppose $h$ passes through a point $q$ in the interior of $e_{i j}(t)$, and let $A(h, t)$ be the dual angle in $h$ at $q$ so that $\gamma_{i j}(h, t)=\alpha A(h, t)$. If $h=h_{0}$ is orthogonal to $e_{i j}(t)$, then $\gamma_{i j}\left(h_{0}, t\right)=\beta_{i j}(t)$, the edge curvature along $e_{i j}(t)$ as defined in $\S 3$.

The key observation is that $A(h, t)$ is the orthogonal projection of $A\left(h_{0}, t\right)$ onto $h$. To see this, observe that an extreme ray in $A\left(h_{0}, t\right)$ is also normal to a facet of $K$. Therefore the projection of this ray onto $h$ will be orthogonal to a side of the interior angle at $q$ in $h$ of $h \cap K$. Thus the projected ray is an extreme ray of the dual angle at $q$ in $h$ of $h \cap K$.

Proceeding to integrate equation (24), using (5), the following equations are immediate since all functions are bounded;

$$
2 \sigma_{n-2} M(K(t))=\int \sum_{i j} \gamma_{i j}(h, t) d \eta(h)=\sum_{i j} \int \gamma_{i j}(h, t) d \eta(h) .
$$

Next fix $i, j$ and consider the equations

$$
\begin{aligned}
\int_{H\left[e_{i j}(t)\right]} \gamma_{i j}(h, t) d \eta(h) & =\iint_{e_{i j}(t) H(q)} \gamma_{i j}(h, t)|\cos \theta(h, t)| d \mu(h) d q \\
& =c_{n} \beta_{i j}(t) l_{i j}(t) .
\end{aligned}
$$

The first equation in (26) is obtained by use of the fundamental differential relation

$$
d \eta(h)=|\cos \theta(h, t)| d \mu(h) d q,
$$

where $\theta(h, t)$ is the angle between the unit normal $n(h)$ and the segment $e_{i j}(t)$, and $d q$ gives linear measure on $e_{i j}(t)$. The verification that the inner integral equals $c_{n} \beta_{i j}(t)$ is an exercise on invariant measures.

Summing (26) over all $i, j$ yields the formula (10), whose derivation was promised earlier.

Before integrating equation (25) we observe that if $q$ is any point in $E^{n}$, equation (25) is valid on any plane in $H(q)$ which does not contain a vertex $p_{i}(t)$; for if $h$ does not cut $K(t), \gamma_{i j}(h, t)=0$, and $(d / d t) \gamma_{i j}(h, t)=0$ is a well-defined statement since "not cutting" is an open property with respect to $t$. It follows at once that (25) is valid a.e. $(\mu)$ and a.e. $(\eta)$. 
The integration of equation (25) with respect to $\eta$ clearly gives zero. However, term-by-term integration leads to the problem of showing that

$$
\begin{aligned}
\int_{H\left[e_{i j}(t)\right]} \frac{d}{d t} \gamma_{i j}(h, t) d \eta(h) & =\iint_{e_{i j}(t) H(q)} \frac{d}{d t} \gamma_{i j}(h, t)|\cos \theta(h, t)| d \mu(h) d q \\
& =c_{n} l_{i j}(h) \frac{d}{d t} \beta_{i j}(t) .
\end{aligned}
$$

The first equality in (28) follows from the previously discussed differential relation (27) provided we can prove integrability. We proceed to analyze the inner integral over $H(q)$,

$$
\int \frac{d}{d t} \gamma_{i j}(h, t)|\cos \theta(h, t)| d \mu(h) .
$$

As in the proof of Theorem 5 , let $\left\{\Delta_{k} t\right\}$ be a sequence of positive numbers tending to zero so that $\sum_{k} \Delta t$ converges. The previous integral equals

$$
\begin{aligned}
\int \operatorname{limit}_{\Delta_{k} t \rightarrow 0} & \frac{\gamma_{i j}\left(h, t+\Delta_{k} t\right)-\gamma_{i j}(h, t)}{\Delta_{k} t}|\cos \theta(h, t)| d \mu(h) \\
& =\operatorname{limit}_{\Delta_{k} t \rightarrow 0} \int \frac{\gamma_{i j}\left(h, t+\Delta_{k} t\right)-\gamma_{i j}(h, t)}{\Delta_{k} t}|\cos \theta(h, t)| d \mu(h) .
\end{aligned}
$$

In explaining equation (29) first note that there is logical meaning to both sides since $\gamma_{i j}(h, t)$ is well defined independently of $q$ due to the translation invariance of the measures involved in defining angle projection onto $h$. This was discussed earlier with regard to equations (14) and (15).

Since $|\cos \theta(h, t)| \leqslant 1$, the function $G^{\prime}=d_{n-1} \sum G$, constructed (see (23)) in $\S 4$, allows application of the dominated convergence theorem to interchange the order of limit and integral. Thus $(d / d t) \gamma_{i j}(h, t)|\cos \theta(h, t)|$ is $\mu$-integrable, and hence $(d / d t) \gamma_{i j}(h, t)$ is $\eta$-integrable according to the theorems of Fubini-Tonelli. More precisely, the function $G^{\prime}(h)$ has an obvious extension to the entire set of planes which cut the interior of $e_{i j}(t)$ (or any planeset, if need be), defined by $G^{\prime}\left(h_{1}\right)=$ $G^{\prime}\left(h_{2}\right)$ for $h_{1}$ parallel to $h_{2}$. Tonelli's theorem may be applied to the nonnegative function $G^{\prime}(h)|\cos \theta(h)|$ to show that $G^{\prime}(h)$ is $\eta$-integrable.

The right side of (29) may be expressed as

$$
\begin{aligned}
\operatorname{limit}_{\Delta t \rightarrow 0} \int \frac{\gamma(h, t+\Delta t)|\cos \theta(h, t+\Delta t)|-\gamma(h, t)|\cos \theta(h, t)|}{\Delta t} d \mu(h) \\
-\operatorname{limit}_{\Delta t \rightarrow 0} \int \frac{|\cos \theta(h, t+\Delta t)|-|\cos \theta(h, t)|}{\Delta t} \gamma(h, t+\Delta t) d \mu(h),
\end{aligned}
$$

provided both limits exist. The subscripts $i, j, k$ are omitted in (30). The first limit in (30) may be evaluated as

$$
\operatorname{limit}_{\Delta t \rightarrow 0} c_{n}\left(\frac{\beta(t+\Delta t)-\beta(t)}{\Delta t}\right)=c_{n} \frac{d \beta}{d t} .
$$

Because $p_{i}(t)$ and $p_{j}(t)$ are smooth functions of $t,|\Delta \theta| \leqslant K|\Delta t|$; and it follows easily that the integrand of the second limit in $(30)$ is bounded. The bounded 
convergence theorem implies that this limit is

$$
\int_{H(q)} \frac{d}{d t}|\cos \theta(h, t)| \cdot \gamma(h, t) d \mu(h) .
$$

If $H_{q}$ is identified with the sphere $S^{n-1}$, it may be noted that the integrand of (31) is antisymmetric with respect to the axis of $S^{n-1}$ parallel to $e_{i j}(t)$, and hence the integral (31) is zero.

The preceding work shows that

$$
\int \frac{d}{d t} \gamma(h, t)|\cos \theta(h, t)| d \mu(h)=c_{n} \frac{d \beta}{d t},
$$

and a simple application of Fubini's theorem verifies the second equality of (28). Summing over all $i, j$ we obtain equation (11). The product rule for differentiation gives equation (12). This completes the proof of Theorem 4.

6. Proofs of Theorem 1 and Theorem 2. For any convex body $K$ in $E^{m}$, $M(c K)=c M(K)$ for $c \geqslant 0$. It follows at once that Theorem 1 is true for all $c$ if it is true for $c=1$. Also, if $\left\{p_{0}, p_{1}, \ldots, p_{n}\right\}$ and $\left\{q_{0}, q_{1}, \ldots, q_{n}\right\}$ are pointsets in $E^{n}$ which satisfy condition (1) for $c=1$, then the points may be moved slightly so that condition (1) remains satisfied, but the pointsets are the vertex sets of two nondegenerate simplices in $E^{n}$. If Theorem 1 holds in this nondegenerate case, then the continuity of $M$ with respect to the Blaschke-Hausdorff metric on compact convex sets in $E^{n}$ shows that Theorem 1 is valid when $m=n$.

To deal with the nondegenerate case of $n+1$ points in $E^{n}$, use Lemma 1 to form for $0<t<1$ a smooth family of simplices $K(t)$ with $K(0)$ congruent to $\operatorname{conv}\left\{q_{i}\right\}$ and $K(1)$ congruent to $\operatorname{conv}\left\{p_{i}\right\}$. By Theorem 3,

$$
\frac{d}{d t} M(K(t))=c_{n} \sum_{i j} \beta_{i j}\left(\frac{d l_{i j}}{d t}\right),
$$

and hence $(d / d t) M(K(t)) \geqslant 0$ because $\beta_{i j}(t) \geqslant 0$ and $(d / d t) l_{i j}(t) \geqslant 0$ for each $i, j$. Thus $M\left(\operatorname{conv}\left\{q_{i}\right\}\right) \leqslant M\left(\operatorname{conv}\left\{p_{i}\right\}\right)$.

Finally, if $M^{\prime}$ and $M$ are the total mean curvature functions on compact convex sets in $E^{m}$ and $E^{n}$, there is a constant $b(m, n)$ such that

$$
M^{\prime}(K)=b(m, n) M(K)
$$

for $K \subset E^{m} \subset E^{n}$. The identity (32) is an easy consequence of formula (4); $b(2,3)=$ $\pi$, for example. Since the case $m \neq n$ now follows at once, the proof of Theorem 1 is complete.

As was indicated earlier, Theorem 2 is a diret consequence of Theorem 1. If, as seems clear, Theorem 4 generalizes to smooth families of convex polyhedra in $E^{n}$, then Theorem 2 becomes a corollary to the special case $n=4$. This is because if the $\left\{p_{i}\right\}$ and $\left\{q_{i}\right\}$ are planar pointsets, the the homotopy (8) occurs entirely in $E^{4}$. A simple inductive argument allows one to assume that for all $0 \leqslant t \leqslant 1, p_{i}(t)$ is an extreme point of $\operatorname{conv}\left\{p_{i}(t)\right\}$. 
7. Polyhedral surfaces in $E^{3}$, Connelly spheres. Let $K(t)$ be a smooth family of simplices in $E^{3}$. There is the simple relation $\beta_{i j}(t)=\pi-\alpha_{i j}(t)$ where $\alpha_{i j}(t)$ is the measure of the dihedral angle along $e_{i j}(t)$. So in $E^{3}$, equation (11) is equivalent to

$$
\sum_{i j} l_{i j}(t) \frac{d}{d t} \alpha_{i j}(t)=0 .
$$

Next suppose that $C$ is a closed polyhedral surface in $E^{3}$, along with its interior, which has been triangulated by nondegenerate 3 -simplices $K_{1}, K_{2}, \ldots, K_{r}$. Suppose that the 0 -skeleton $v_{1}, v_{2}, \ldots, v_{s}$ is smoothly deformed for $0 \leqslant t \leqslant 1$ as $\left\{v_{1}(t), \ldots, v_{s}(t)\right\}$, so that for each $t, K_{1}(t), \ldots, K_{r}(t)$ is a triangulation by nondegenerate 3-simplices of a closed polyhedral surface with interior $C(t)$; set $C=C(0)$.

Equation (33) is valid in each simplex $K_{k}(t)$ so that the sum over $C(t)$ is zero. However, if $e_{i j}(t)$ is an element of the 1-skeleton which, except possibly for $v_{i}(t)$ or $v_{j}(t)$, lies in the interior of $C(t)$, we have $\Sigma_{k}\left\{\alpha_{i j k}(t): e_{i j} \in K_{k}(t)\right\}=2 \pi$. Hence $\sum_{k} l_{i j}(d / d t) \alpha_{i j k}=0$. Therefore if we sum only those terms $l_{i j}(d / d t) \alpha_{i j k}$ where $e_{i j}(t)$ lies in $\partial C(t)$ the result is still zero.

If $e_{i j}$ lies on $\partial C, \beta_{i j}=\pi-\alpha_{i j}$, where $\alpha_{i j}$ measures the dihedral angle along $e_{i j}$ interior to $\partial C$. Define $M(\partial C)=\sum\left\{l_{i j} \beta_{i j}: e_{i j} \subset \partial C\right\}$. (This agrees with the formula given by Blaschke [3].)

The previous observations immediately give the following theorem.

THEOREM 6. Let $C(t)$ be a smoothly deformed polyhedral surface in $E^{3}$ (as described above). Then for $e_{i j} \subset \partial C(t)$,

$$
\sum_{i j} l_{i j}(t) \frac{d}{d t} \beta_{i j}(t)=0,
$$

and hence

$$
\frac{d}{d t} M(\partial C(t))=\sum_{i j} \beta_{i j}(t) \frac{d}{d t} l_{i j}(t) .
$$

As we remarked in the Introduction, Connelly [6] has given ingenious constructions of embedded polyhedral 2-spheres in $E^{3}$ which flex, i.e., may be deformed in a manner which causes no nontrivial deformation of any 2-face. It is easily seen that there is no loss of generality in assuming that all 2-faces are triangles.

In a flex, $(d / d t) l_{i j}(t) \equiv 0$ for all $i, j$ in $(35)$, hence $(d / d t) M(\partial C) \equiv 0$ which implies $M(\partial C)$ is constant during the flex. We restate Theorem 6 in a slightly expanded form, although Connelly spheres are the only known embedded closed flexible polyhedral surfaces. No example of positive genus has been discovered.

TheOrem 3'. Any flexible closed polyhedral surface in $E^{3}$ must flex with constant total mean curvature.

Also, it might be observed that for a flexible polyhedral surface, repeated differentiation of (34) yields

$$
\sum_{i j} l_{i j} \frac{d^{r}}{d t^{r}} \beta_{i j}=0, \quad r \geqslant 1 .
$$


8. Extended versions of Theorem 4 and Theorem 5. Let $H_{r}(q), 1 \leqslant r \leqslant n-1$, be the manifold of oriented $r$-flats which pass through the point $q$ in $E^{n}$. There is a natural invariant measure $\mu_{r}$ on $H_{r}(q)$. The structure of $\mu_{r}$ as well as that of $\eta_{r}$, the invariant measure on the oriented $r$-flats of $E^{n}$, is discussed by Santaló in Chapter 12 of his book [11]. If $1 \leqslant k \leqslant r$, let $A(t)$ denote a smooth family of $k$-angles, and let $A(h, t)$ denote the orthogonal projection of $A(t)$ onto the $r$-plane $h$. The ideas used to prove Theorem 5 may be applied without severe modification to yield the following extension. Here $\alpha$ represents $(k-1)$-measure on $S^{k-1}$.

TheOREM 5. $d / d t \alpha A(t)=c(n, r, k) \int_{H_{r}(q)} d / d t \alpha A(h, t) d \mu_{r}(h)$.

Next, if $K=\operatorname{conv}\left\{p_{0}, \ldots, p_{n}\right\}$ is a simplex in $E^{n}$, let $V_{j}, j=\left\{i_{0}, i_{1}, \ldots, i_{n-s}\right\}$, denote the $(n-s)$ volume of $\operatorname{conv}\left\{p_{i}: i \in j\right\}$, and let $\beta_{j}$ denote the measure of the dual angle (an $s$-angle) at $V_{j}$. The extreme rays of $\beta_{j}$ are the normals of the $s$ $(n-1)$-faces of $K$ whose intersection is $V_{j}$. Clearly $j$ ranges over $\left(\begin{array}{l}n \\ s\end{array}\right)$ indices. Corresponding to the formula (10) there is the following formula for $C_{s}(K)$, the $\eta_{s}$-measure of the $s$-planes which cut $K$ :

$$
C_{s}(K)=c(n, s) \sum_{j} V_{j} \beta_{j} \text {. }
$$

THEOREM 4'. Let $K(t)$ be a smooth family of simplices in $E^{n}$, and suppose $2 \leqslant s \leqslant n-1$. Then for those $j$ with $|j|=n-s$,

$$
\sum_{j} V_{j}(t) \frac{d \beta_{j}}{d t}=0
$$

and hence

$$
\frac{d}{d t} C_{s}(K(t))=c(n, s) \sum_{j} \beta_{j}(t) \frac{d V_{j}}{d t} .
$$

Proof (Sketch). Of those $s$-planes $h$ which cut $K(t)$, almost all $\left(\eta_{s}\right)$ have the property that $h \cap K(t)$ is an $s$-polytope, all of whose vertices lie in the interiors of various $(n-s)$ faces of $K(t)$. Equation (25), with $j$ replacing $i j$, may be integrated with respect to $\eta_{s}$. At a given point $q$ in the interior of $V_{j}$ we have an analogue of the differential (27): for $s \leqslant n-s, d \eta_{s}=c(n, s)\left|\cos \theta_{1} \cdots \cos \theta_{s}\right| d \mu_{s} d q$ where $d q$ integrates to $(n-s)$ measure on $V_{j}$ and $\left|\cos \theta_{1} \cdots \cos \theta_{s}\right|$ is the Jacobian of the orthogonal projection mapping from the $s$-plane $h$ to $V_{j}$. If $s>n-s$, the Jacobian takes the form $\cos \theta_{1} \cdots \cos \theta_{n-s}$. The angle $\theta_{1}$ is the minimal angle between $h$ and $V_{j}$, etc. Equation (38) is validated by termwise integration of (25). A truly selfcontained discussion laying out all details, as in the previous proof for $s=n-1$, will be appropriate for $2<s<n-1$ when there are more applications. It is hoped that (39) will be useful in the study of higher order discrete integral mean curvature as well as volume inequalities.

The case $s=2$, which agrees with $s=n-1$ for the important special case $n=3$, has several direct proofs. Two such proofs are given in $\S 9$, and thus our observations about Connelly spheres will not depend on Theorem 5 . 
9. Two direct proofs for the case $s=2$; Schläfli's differential formula. Let $K(t)$ be a smooth family of $n$-simplices in $E^{n}$, and let $G(t)$ be an $(n-2)$-face of $K(t)$. Let $n(t)$ and $n^{\prime}(t)$ be the outward pointing unit normals to the $(n-1)$-faces of $K(t)$, $F(t)$ and $F^{\prime}(t)$, that have $G(t)$ as a common face. Let $v(t)$ and $v^{\prime}(t)$ be vectors in the hyperplanes of $F(t)$ and $F^{\prime}(t)$, respectively, that are outernormal to $G(t)$, and such that

$$
|v(t)|=\left|v^{\prime}(t)\right|=\operatorname{Vol} G(t)
$$

Then

$$
-\operatorname{Vol} G(t)\left(\frac{d \beta}{d t}\right)=\left\langle\frac{d n}{d t}, v(t)\right\rangle+\left\langle\frac{d n^{\prime}}{d t}, v^{\prime}(t)\right\rangle,
$$

where $\beta(t)$ is the angle between $n(t)$ and $n^{\prime}(t)$. Equations (40) can be seen for instance by writing $n, n^{\prime}, v, v^{\prime}$ in a coordinate system for a 2-plane which is orthogonal to the $(n-2)$-face $G$. (This is illustrated in Figure 1$)$. Then

$$
\begin{gathered}
n(t)=\cos \theta(t) n_{1}+\sin \theta(t) n_{2}, \\
n^{\prime}(t)=\cos \theta(t) n_{1}+\sin \theta^{\prime}(t) n_{2}, \\
v(t)=\operatorname{Vol} G(t)\left[\sin \theta(t) n_{1}-\cos \theta(t) n_{2}\right], \\
v^{\prime}(t)=\operatorname{Vol} G(t)\left[-\sin \theta^{\prime}(t) n_{1}+\cos \theta^{\prime}(t) n_{2}\right] .
\end{gathered}
$$

Clearly $\beta(t)=\theta^{\prime}(t)-\theta(t)$; formula (40) follows from a direct calculation.

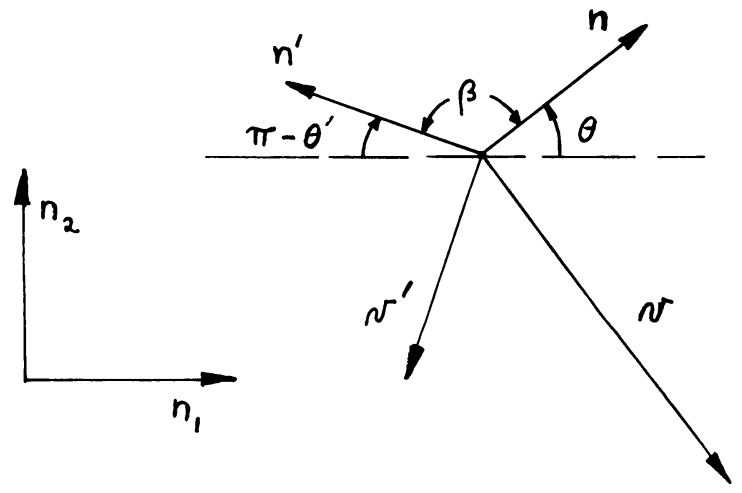

Figure 1

For each $F(t)$ let $v_{i}(t), i=0, \ldots, n-2$, be the outward pointing normal to the $(n-2)$-faces of $F(t)$, with length equal to the $(n-2)$-volume of the corresponding $(n-2)$-face. The well-known observation of Minkowski that $\int n d \sigma=0$, where $n$ is the outer normal and the integral is over a closed surface, shows $\sum_{i=0}^{n-2} v_{i}(t)=0$.

Summing over the various $(n-1)$-faces $F_{j}$ gives $\sum_{j} \Sigma_{i}\left\langle d n_{j} / d t, v_{i j}(t)\right\rangle=0$, and substituting (40) for each pair of terms corresponding to the various $(n-2)$-faces of $K(t)$ gives formula (38) for $s=2$.

It may also be noted that this derivation shows that a natural smooth version of (38) for $s=2$ is $\int \operatorname{div}(d n / d t) d \sigma=0$, where $n(t)$ is unit outer normal to a smooth family of smooth closed hypersurfaces in $\mathbf{E}^{n}$. This smooth identity is an immediate 
consequence of Stoke's theorem. Chapter 12 of Spivak's book [12] discusses the basic results on the rigidity of smooth surfaces. Though a discussion would take us too far off course, we remark that the Herglotz integral formula can be used to show that a smooth analogue of Theorem 3 does hold. However, no example of an embedded closed bendable smooth surface is known.

The second direct proof is not self-contained in that Schläfli's differential formula is employed. However, in the sequel (Part II) this formula will be derived as an application of the methods in the two articles. The book of Böhm and Hertel [4] contains a detailed analytic derivation of the formula.

If $K^{\prime}(t)$ is a smooth family of simplices on $S^{n}$, then Schläfli's formula says that for $|j|=n-2$

$$
\frac{d}{d t} \operatorname{Vol} K^{\prime}(t)=-\frac{1}{2(n-1)} \sum_{j} V_{j}^{\prime}(t) \frac{d}{d t} \beta_{j}^{\prime}(t)
$$

Here $\beta_{\mathrm{j}}^{\prime}$ and $V_{j}^{\prime}$ on $S^{n}$ are analogous to $\beta_{j}$ and $V_{j}$ in $E^{n}$. If $K^{\prime}(t)$ lies on an $n$-sphere of radius $\rho$, dimensional considerations show that the right side of (41) must be multiplied by $\rho^{2}$.

If $K(t)$ is a smooth family of simplices in $E^{n}$, choose a point $q$ in $E^{n}$ and an $n$-sphere $T$ in $E^{n+1}$ which is tangent to $E^{n}$ at $q$. Let $K^{\prime}(t)$ be the smooth family on $T$ obtained by radially projecting $K(t)$ onto $T$. It is clear that as $\rho \rightarrow \infty$, $(d / d t) \operatorname{Vol} K^{\prime}(t)$ and $(d / d t) \beta_{j}^{\prime}(t)$ are of the same order of magnitude as $(d / d t) \operatorname{Vol} K(t)$ and $(d / d t) \beta_{j}(t)$, respectively. Thus for some constant $c$,

$$
c\left|\frac{d \mathrm{Vol} K}{d t}\right| \geqslant \rho^{2}\left|\sum V_{j} \frac{d \beta_{j}}{d t}\right|, \quad|j|=n-2 .
$$

Letting $\rho \rightarrow \infty$ again leads to formula (38) for $s=2$.

10. A simple example in $E^{3}$. Let the smooth family $K(t)$ be given by $p_{0}=(0,0,0)$; $p_{1}=(1,0,0) ; p_{2}=(0,1,0) ; p_{3}=(0,0, t), t>0$. It follows that

$$
\begin{gathered}
n_{0}=\left(2 t^{2}+1\right)^{-1 / 2}(t, t, 1) ; \quad n_{1}=(-1,0,0) ; \\
n_{2}=(0,-1,0) ; \quad n_{3}=(0,0,-1) .
\end{gathered}
$$

There are three distinct nonzero terms in formula (11) in this situation. The formula $\left\langle n_{i}, n_{j}\right\rangle=\cos \beta_{i j}$ gives a desired formula. However, it turns out that conversion to $\tan \beta_{i j}$ generally gives simpler expressions. Summing gives

$$
\begin{aligned}
l_{12} \frac{d}{d t} \beta_{12}+ & l_{23} \frac{d}{d t} \beta_{23}+l_{31} \frac{d}{d t} \beta_{31} \\
= & \sqrt{2} \frac{d}{d t} \tan ^{-1}(-\sqrt{2} t)+\sqrt{1+t^{2}} \frac{d}{d t} \tan ^{-1}\left(-t^{-1} \sqrt{1+t^{2}}\right) \\
& +\sqrt{1+t^{2}} \frac{d}{d t} \tan ^{-1}\left(-t^{-1} \sqrt{1+t^{2}}\right) \\
= & -2\left(1+2 t^{2}\right)^{-1}+\left(1+2 t^{2}\right)^{-1}+\left(1+2 t^{2}\right)^{-1}=0,
\end{aligned}
$$

as predicted by Theorem 4 . 
A simple further calculation shows that $\sum l_{i j}\left(d^{2} / d t^{2}\right) \beta_{i j}$ is not zero. This example shows that there is no obvious generalization of Theorem 5 involving higher derivatives of the projected angle since the resulting generalization of Theorem 4 is false. Thus equation (36) says something special about flexible polyhedral surfaces.

11. Further problems and observations. Branko Grünbaum asks whether there are any two-dimensional linear spaces other than $E^{2}$ for which Theorem 2 is valid. We note that with the strong extra assumption that all $p_{i}$ lie on the boundary of $\operatorname{conv}\left\{p_{i}\right\}$, the conclusion is valid. This follows at once from the fact that for any two-dimensional linear space there is a measure $\eta$ on the linesets such that $\|p-q\|=$ $\frac{1}{2} \eta\{l: l \cap \bar{p} \bar{q} \neq \varnothing\}$. (See G. D. Chakerian's paper [5].) The general question remains open even for four-point sets.

Assuming $c=1$ in the Lipschitz condition (1), G. D. Chakerian asks whether Theorem 2 remains valid on $S^{n}$. Fortunately, this question can now be answered affirmatively. If $K(t)$ is a smooth family of simplices on $S^{n}$, let $C(t)$ denote the invariant measure of the great $(n-1)$-spheres which cut $K(t)$. As a consequence of Schläfli's differential formula (41) the following relation holds via duality:

$$
\frac{d}{d t} C(t)=c_{n} \sum \beta_{i j}(t) \frac{d}{d t} l_{i j}(t)
$$

Thus there is a direct analogy with formula (12). Further investigation will be required to determine the spherical (and hyperbolic) analogues of formulas (11), (38), (39). There will be a complete discussion of equation (42) in the sequel.

Let $D_{0}, \ldots, D_{s}$ and $D_{0}^{\prime}, \ldots, D_{s}^{\prime}$ be disks of fixed radius $R$ in $\mathbf{E}^{2}$ with centers $p_{0}, \ldots, p_{s}$ and $p_{0}^{\prime}, \ldots, p_{s}^{\prime}$, respectively. Klee $[10]$ asks whether the condition $\left|p_{i}-p_{j}\right| \leqslant$ $\left|p_{i}^{\prime}-p_{j}^{\prime}\right|$, all $i, j$, ensures that Area $\cap D_{i} \geqslant$ Area $\cap D_{i}^{\prime}$. We ask if the stronger result, Per $\cap D_{i} \geqslant \operatorname{Per} \cap D_{i}^{\prime}$, must also follow. This question is pursued a bit further in the next paragraphs.

Let us assume that $\partial D_{j} \cap\left(\cap D_{i}\right) \neq \varnothing$ for $j=0, \ldots, s$, and let $E=\partial\left(\cap D_{i}\right)$. The centers $p_{0}, \ldots, p_{s}$ are the vertices of the evolute of $E$, denoted by $E^{*}$. It is easily seen that $E^{*}=\partial\left(\cap D_{i}^{*}\right)$ where $D_{0}^{*}, \ldots, D_{s}^{*}$ are disks of radius $R$ centered at the vertices of $E$, and that $E^{* *}=E$. There is the relation

$$
\text { Length } E+\text { Length } E^{*}=2 \pi R \text {. }
$$

We therefore see that our question concerning $\operatorname{Per} \cap D_{i}$ is equivalent to the question: if $\left|p_{i}-p_{j}\right| \leqslant\left|p_{i}^{\prime}-p_{j}^{\prime}\right|$ for all $i, j$, is it true that Length $E^{*} \leqslant \operatorname{Length}\left(E^{\prime}\right)^{*}$ ? The difficulty arises because generally there will be $j$ for which $\partial D_{j}^{\prime} \cap\left(\cap D_{i}^{\prime}\right)=\varnothing$.

Now if $R$ becomes large while keeping $\left\{p_{i}\right\}$ and $\left\{p_{i}^{\prime}\right\}$ fixed, the question on Length $E^{*}$ becomes precisely the question answered by Theorem 2 . This establishes a direct link between Klee's question and the results in this paper. There are generalizations of (43) in higher dimensions which might lead to further conjectures or counterexamples.

Let $q_{0}, \ldots, q_{n}$ be points in $\mathbf{E}^{2}$. Define a partial reflection to be a line reflection of a subset of the $q_{i}$ through a line which does not enter the interior of $\operatorname{conv}\left\{q_{i}\right\}$. The 
complement remains fixed. Suppose $\left\{q_{i}\right\}$ and $\left\{p_{i}\right\}$ sastisfy the Lipschitz condition (1) with $c=1$. Will there always be a (finite) sequence of partial reflections and continuous distance nondecreasing planar homotopies taking $q_{i}$ to $p_{i}$ for each $i$ ? A case analysis shows that the answer is affirmative for four-point sets.

R. V. Ambartzumian [2] has developed a very interesting combinatorial theory of mean curvature and generalized Crofton measures. Do any of the results of this article fit into the framework of his theory?

ACKNowledgment. We wish to thank the Institut des Hautes Études Scientifiques for their kind hospitality during the spring of 1980 when some of the ideas in this paper were first formulated. Also, we are grateful for the kind interest and helpful suggestions of S. B. Alexander, R. Connelly, G. D. Chakerian, and B. Grünbaum. Credit for the first proof in $\$ 9$ is shared with R. Jerrard. Finally, thanks are due to the referee for a number of suggestions which improved the accuracy and readability of the article.

\section{REFERENCES}

1. R. Alexander, The circumdisk and its relation to the theorem of Kirszbraun and Valentine, Math. Mag. 57 (1984), 165-169.

2. R. V. Ambartzumian, On some topological invariants in integral geometry, Z. Wahrsch. Verw. Gebiete 44 (1978), 57-69.

3. W. Blaschke, Vorlesungen über Integralgeometrie, 3rd ed., Deutsch. Verlag Wiss., Berlin, 1955.

4. J. Böhm and E. Hertel, Polyedergeometrie in n-dimensionalen Räumen konstanter Krümmung, Birkhäuser Verlag, Basel, 1981.

5. G. D. Chakerian, Integral geometry in the Minkowski plane, Duke Math. J. 29 (1962), 375-381.

6. R. Connelly, A counterexample to the rigidty conjecture for polyhedra, Publ. Math. Inst. Hautes Études Sci. 47 (1977), 333-338.

7. W. J. Firey, Christoffel's problem for general convex bodies, Mathematika 15 (1968), 7-21.

8. B. Grünbaum, Convex polytopes, Wiley, New York, 1967.

9. M. Kirszbraun, Über die zusammenziehenden und Lipschitzschen Transformationen, Fund. Math. 22 (1934), 77-108.

10. V. Klee, Some unsolved problems in plane geometry, Math. Mag. 52 (1979), 131-145.

11. L. A. Santalo, Integral geometry and geometric probability, Encyclopedia of Mathematics and its Applications, Vol. I, Addison-Wesley, Reading, Mass., 1976.

12. M. Spivak, A comprehensive introduction to differential geometry, Publish or Perish, Berkeley, Calif., 1979.

Department of Mathematics, 1409 West Green Street, University of Illinois, Urbana, ILLINOIS 61801 